\title{
Genetic Diversity and Distribution of Korean Isolates of Ralstonia solanacearum
}

Yeonhwa Jeong, Jinwoo Kim, and Yongsung Kang, Department of Agricultural Biotechnology and Center for Agricultural Biomaterials, Seoul National University, Seoul 151-921, Korea; Seungdon Lee, Plant Pathology Division, National Institute of Agricultural Science of Technology, Rural Development Administration, Suwon 441-707, Korea; and Ingyu Hwang, Department of Agricultural Biotechnology and Center for Agricultural Biomaterials, Seoul National University, Seoul 151-921, Korea

\begin{abstract}
Jeong, Y., Kim, J., Kang, Y., Lee, S., and Hwang, I. 2007. Genetic diversity and distribution of Korean isolates of Ralstonia solanacearum. Plant Dis. 91:1277-1287.

Genetic diversity among 478 isolates of Ralstonia solanacearum collected from various plants in Korea between 1997 and 2005 was determined based on biovar, pathogenicity, amplified fragment length polymorphism (AFLP), 16S rRNA, endoglucanase, $h r p B$, and mutS gene sequence analyses. Of the isolates, 440 belonged to biovars 1,3 , or 4 , and 38 belonged to biovar 2 . Biovar $\mathrm{N} 2$ isolates were not found. The biovar 1 and 2 isolates were found mainly in southern Korea, whereas the biovar 3 and 4 isolates were widely distributed throughout all nine provinces. AFLP analysis divided the 109 representative Korean isolates into six clusters that were distinct from most of the foreign isolates. Grouping of 8 representative isolates based on their 16S rRNA gene sequences indicated that biovars 1,3 , and 4 belonged to division 1 , while biovar 2 belonged to subdivision $2 \mathrm{~b}$. Sequence analysis of the endoglucanase, $h r p B$, and $m u t S$ genes from the same isolates indicated that the biovar 1, 3, and 4 isolates belonged to phylotype I, while the biovar 2 isolate belonged to phylotype IV. This study is the first comprehensive analysis of genetic diversity among Korean isolates of $R$. solanacearum.
\end{abstract}

Bacterial wilt caused by Ralstonia solanacearum is one of the most important bacterial plant diseases in the world's tropical, subtropical, and warm temperate regions (10). The bacterium infects many economically important crops, including tomato (Lycopersicon esculentum Mill.), potato (Solanum tuberosum L.), pepper (Capsicum annuum L.), tobacco (Nicotiana tabacum L.), eggplant (Solanum melongena L.), banana (Musa paradisiaca L.), ginger (Zingiber officinale Roscoe), and peanut (Arachis hypogaea L.) (10).

The species $R$. solanacearum is a complex taxonomic unit with broad physiological and genetic diversity; therefore, it has been divided into seven biovars (based on biochemical properties) and five races (based on differences in host range) $(10,11)$. Unlike other phytopathogenic bacteria, race systems of $R$. solanacearum are not based on gene-for-gene interactions (i.e., different cultivars carrying different $R$ gene[s]). Instead, they are based on the pathogenicity of each isolate in different kinds of host plants $(3,10)$. Although the biovar and race systems are widely accepted for the classification of $R$. solana-

Corresponding author: Ingyu Hwang

E-mail: ingyu@snu.ac.kr

Accepted for publication 9 May 2007.

doi:10.1094/PDIS-91-10-1277

(C) 2007 The American Phytopathological Society cearum, there is no definite correlation between biovar and race: each race transects the biovars and each biovar contains various races $(9,10,11)$. The only positive correlation between the biovar and race systems exists for biovar 2 and race $3(10,26)$.

To circumvent such classical taxonomic methods and improve our understanding of the genetic relatedness among $R$. solanacearum strains, DNA-based analyses such as restriction fragment length polymorphisms (RFLP), polymerase chain reaction (PCR)-RFLP, 16S rRNA gene sequence analysis, repetitive extragenic palindromic (REP)-PCR, and amplified fragment length polymorphism (AFLP) are now used $(6,8,13,21)$. RFLP analysis of $R$. solanacearum isolates divided the species into two major groups that reflected their geographical origins $(4,5,6)$. The first group, called the Americanum division, contains biovar 1, 2, and N2 isolates, while the second group, the Asiaticum division, contains biovar 3,4 , and 5 isolates $(4,5,6)$. Further analysis by $16 \mathrm{~S}$ rRNA gene sequencing confirmed the results of the RFLP analysis and separated division 2 into subdivisions, which included the Indonesian isolates $(19,21,25,27)$. Subgroup specific primers were designed using the sequences of the $16 \mathrm{~S}-23 \mathrm{~S}$ intergenic spacer region of the rRNA gene, the polygalacturonase gene, and the endoglucanase gene (8), but the resulting classification system was not fully consistent with the results of a PCR-RFLP analysis based on the hrp gene in many African biovar 1 isolates that assigned them to the Asiaticum division (22). REP-PCR analysis using different primer sets with $16 \mathrm{~S}$ rRNA gene sequence analysis yielded the genomic fingerprints of several Japanese strains $(12,13)$; however, the grouping obtained by this method failed to reflect the diverse hosts and geographical origins from which the isolates were obtained. Therefore, AFLP analysis has been used as an alternative DNA fingerprinting tool to classify $R$. solanacearum isolates at a subspecies level $(21,32)$. AFLP-based analysis of $R$. solanacearum isolates collected worldwide (e.g., African, American, and Japanese) produced seven clusters reflecting the biovars and their geographical origins (21).

In 2005, a new hierarchical classification scheme based on sequence analyses of the endoglucanase, $h r p B$, and $m u t S$ genes was proposed $(7,23)$. This new scheme divided the $R$. solanacearum species complex into four phylotypes. The isolates in phylotype I originate in Asia, whereas those in phylotype II are primarily from America. Phylotype III mainly comprises isolates from Africa and its surrounding islands, while those in phylotype IV are from Indonesia, Japan, and Australia (7). Phylotypes can be subgrouped into sequevars, which are groups of isolates with highly conserved DNA sequences (7).

Bacterial wilt is a serious and chronic disease in tomato, potato, tobacco, eggplant, and pepper plants during the growing season in Korea, when the temperature and moisture levels are high (31); however, the genetic diversity among Korean $R$. solanacearum isolates is unknown. Thus, we investigated the genetic diversity of Korean isolates of $R$. solanacearum to create a race and biovar distribution map and improve the management of bacterial wilt. Between 1997 and 2005, we collected 478 Korean isolates of $R$. solanacearum and 16 foreign isolates from diverse geographical locations and from different hosts during the growing season. Then, after determining the biovars and races of all 478 isolates, we analyzed 125 representative isolates by AFLP and determined the $16 \mathrm{~S}$ rRNA, endoglucanase, $h r p B$, and $m u t S$ gene sequences for 8 representative isolates. We found that the Korean isolates comprised six AFLP clusters that were distinct from the foreign isolates, and our 
16S rRNA, endoglucanase, $h r p B$, and mutS gene sequence analyses revealed two divisions and two phylotypes.

\section{MATERIALS AND METHODS}

Collection of isolates and culture conditions. Four hundred and seventy-eight $R$. solanacearum isolates were collected from wilted tomato, pepper, eggplant, potato, perilla, sesame, paprika, peanut, pimiento, and sunflower plants at 90 different locations in 9 Korean provinces (Gangwon-do, Gyeonggi-do, Chungcheongbuk-do, Chungcheongnam-do, Jeollabuk-do, Jeollanam- do, Gyeongsangbuk-do, Gyeongsangnamdo, and Jeju-do) between 1997 and 2005 The 109 representative isolates used for further analysis are listed in Table 1. All of the isolates were purified on tetrazolium chloride (TZC) agar medium, frozen in $20 \%$ glycerol and stored at

Table 1. Geographical origin, biovar, race, and amplified fragment length polymorphism (AFLP) group of Ralstonia solanacearum isolates used in this study

\begin{tabular}{|c|c|c|c|c|c|c|}
\hline Isolate $^{x}$ & Geographical origin & Host & Biovar & Race & AFLP cluster & Source ${ }^{y}$ \\
\hline SL312 & Gwangju, Gyeonggi-do & Tomato & 3 & 1 & III & This study \\
\hline SL341 & Gimcheon, Gyeongsangbuk-do & Tomato & 3 & 1 & III & This study \\
\hline SL535 & Jincheon, Chungcheongbuk-do & Tomato & 4 & 1 & III & This study \\
\hline SL776 & Goseong, Gyeongsangnam-do & Tomato & 3 & 1 & III & This study \\
\hline SL1020 & Suwon, Gyeonggi-do & Tomato & 4 & 1 & III & This study \\
\hline SL1085 & Cheorwon, Gangwon-do & Tomato & 4 & 1 & III & This study \\
\hline SL1737 & Boseong, Jeollanam-do & Tomato & 4 & 1 & III & This study \\
\hline SL1743 & Haman, Gyeongsangnam-do & Tomato & 4 & 1 & III & This study \\
\hline SL1971 & Suwon, Gyeonggi-do & Tobacco & 3 & 1 & VI & This study \\
\hline SL1977 & Pohang, Gyeongsangbuk-do & Tobacco & 3 & 1 & VI & This study \\
\hline SL2025 & Boryeong, Chungcheongnam-do & Sesame & 3 & 1 & I & This study \\
\hline SL2029 & Namjeju, Jeju-do & Potato & 2 & 3 & $\mathrm{~V}$ & This study \\
\hline SL2064 & Namjeju, Jeju-do & Potato & 2 & 3 & $\mathrm{~V}$ & This study \\
\hline SL2078 & Namjeju, Jeju-do & Potato & 2 & 3 & $\mathrm{~V}$ & This study \\
\hline SL2249 & Namjeju, Jeju-do & Potato & 1 & 1 & I & This study \\
\hline SL2268 & Namjeju, Jeju-do & Potato & 2 & 3 & $\mathrm{~V}$ & This study \\
\hline SL2282 & Namjeju, Jeju-do & Potato & 2 & 3 & $\mathrm{~V}$ & This study \\
\hline SL2308 & Damyang, Jeollanam-do & Tomato & 2 & 3 & $\mathrm{~V}$ & This study \\
\hline SL2313 & Namhae, Gyeongsangnam-do & Potato & 2 & 3 & $\mathrm{~V}$ & This study \\
\hline SL2316 & Miryang, Gyeongsangnam-do & Potato & 2 & 3 & $\mathrm{~V}$ & This study \\
\hline SL2327 & Namhae, Gyeongsangnam-do & Potato & 4 & 1 & I & This study \\
\hline SL2665 & Boseong, Jeollanam-do & Potato & 2 & 3 & $\mathrm{~V}$ & This study \\
\hline SL2673 & Boseong, Jeollanam-do & Potato & 2 & 3 & $\mathrm{~V}$ & This study \\
\hline SL2680 & Boseong, Jeollanam-do & Potato & 2 & 3 & $\mathrm{~V}$ & This study \\
\hline SL2707 & Gimhae, Gyeongsangnam-do & Potato & 2 & 3 & $\mathrm{~V}$ & This study \\
\hline SL3112 & Namhae, Gyeongsangnam-do & Potato & 2 & 3 & $\mathrm{~V}$ & This study \\
\hline SL3179 & Bukjeju, Jeju-do & Potato & 2 & 3 & $\mathrm{~V}$ & This study \\
\hline SL3202 & Namjeju, Jeju-do & Tomato & 2 & 3 & $\mathrm{~V}$ & This study \\
\hline SL3291 & Namjeju, Jeju-do & Potato & 2 & 3 & $\mathrm{~V}$ & This study \\
\hline SL3505 & Changnyeong, Gyeongsangnam-do & Pepper & 4 & 1 & I & This study \\
\hline EW4 & Hongcheon, Gangwon-do & Eggplant & 3 & 1 & II & This study \\
\hline EW7 & Geoje, Gyeongsangnam-do & Eggplant & 4 & 1 & II & This study \\
\hline EW9 & Gimhae, Gyeongsangnam-do & Eggplant & 4 & 1 & II & This study \\
\hline PW5 & Yanggu, Gangwon-do & Pepper & 3 & 1 & II & This study \\
\hline PW16 & Hampyeong, Jeollanam-do & Pepper & 4 & 1 & II & This study \\
\hline PW17 & Cheongdo, Gyeongsangbuk-do & Pepper & 4 & 1 & I & This study \\
\hline PW22 & Jeongeup, Jeollabuk-do & Pepper & 4 & 1 & II & This study \\
\hline PW23 & Gochang, Jeollabuk-do & Pepper & 4 & 1 & II & This study \\
\hline PW33 & Chilgok, Gyeongsangbuk-do & Pepper & 4 & 1 & I & This study \\
\hline PW40 & Yeonggwang, Jeollanam-do & Pepper & 3 & 1 & II & This study \\
\hline PW44 & Chuncheon, Gangwon-do & Pepper & 4 & 1 & II & This study \\
\hline PAW1 & Hwasun, Jeollanam-do & Paprika & 4 & 1 & VI & This study \\
\hline PAW2 & Tongyeong, Gyeongsangnam-do & Paprika & 4 & 1 & VI & This study \\
\hline POW1 & Yeonggwang, Jeollanam-do & Potato & 4 & 1 & II & This study \\
\hline POW2 & Yeonggwang, Jeollanam-do & Potato & 4 & 1 & II & This study \\
\hline POW3 & Yeonggwang, Jeollanam-do & Potato & 4 & 1 & II & This study \\
\hline POW4 & Yeonggwang, Jeollanam-do & Potato & 4 & 1 & II & This study \\
\hline POW5 & Yeonggwang, Jeollanam-do & Potato & 4 & 1 & II & This study \\
\hline POW7 & Muan, Jeollanam-do & Potato & 4 & 1 & II & This study \\
\hline POW8 & Muan, Jeollanam-do & Potato & 4 & 1 & II & This study \\
\hline POW9 & Muan, Jeollanam-do & Potato & 4 & 1 & II & This study \\
\hline POW10 & Muan, Jeollanam-do & Potato & 4 & 1 & II & This study \\
\hline POW11 & Boseong, Jeollanam-do & Potato & 4 & 1 & II & This study \\
\hline POW12 & Boseong, Jeollanam-do & Potato & 4 & 1 & II & This study \\
\hline POW13 & Boseong, Jeollanam-do & Potato & 4 & 1 & II & This study \\
\hline POW14 & Boseong, Jeollanam-do & Potato & 4 & 1 & II & This study \\
\hline POW15 & Boseong, Jeollanam-do & Potato & 4 & 1 & I & This study \\
\hline POW16 & Boseong, Jeollanam-do & Potato & 4 & 1 & I & This study \\
\hline POW23 & Yeongam, Jeollanam-do & Potato & 4 & 1 & II & This study \\
\hline POW25 & Yeongam, Jeollanam-do & Potato & 4 & 1 & II & This study \\
\hline POW29 & Yeongam, Jeollanam-do & Potato & 4 & 1 & II & This study \\
\hline \multicolumn{7}{|c|}{ (Continued on next page) } \\
\hline
\end{tabular}

${ }^{x}$ Of 494 isolates, 125 representative samples were selected for AFLP analysis based on their geographical origin, biovar, race, and pathogenicity.

y BCCM/LMG: Belgian Coordinated Collection of Microorganisms.

${ }^{\mathrm{z}} \mathrm{N}$ indicates not included in the AFLP clusters. 
$-70^{\circ} \mathrm{C}$ until use (17). The isolates were identified by sole carbon source utilization analysis and fatty acid methyl ester analysis as described previously (15). Of the 16 foreign isolates, 11 were obtained from the Belgian Coordinated Collection of Microorganisms (BCCM/LMG, Ghent, Belgium), 4 were obtained from Kyushu University in Japan, and the GMI1000 isolate was provided by C. Boucher (Table 1).

Pathogenicity and host range tests. For the pathogenicity test, seedlings of tomato (cv. Kwangsoo), pepper (cv. Nokkwang), and potato (cv. Daeji) were transplanted into plastic pots $(11 \mathrm{~cm}$ diameter) (Hungnong Seeds, Seoul, Korea) containing commercial peat mix soil
(Baroker, Seoul Agriculture Materials Co., Seoul, Korea) and grown in a greenhouse at 25 to $30^{\circ} \mathrm{C}$. Each plant was inoculated at the fifth to sixth true leaf stage, and each inoculation was repeated three times. To prepare the inoculum, bacterial cells were grown on TZC agar medium for $24 \mathrm{~h}$ at $28^{\circ} \mathrm{C}$, suspended in sterile distilled water, and adjusted to $10^{8} \mathrm{CFU} / \mathrm{ml}$. The roots of

Table 1. (Continued from previous page)

\begin{tabular}{|c|c|c|c|c|c|c|}
\hline Isolate $^{x}$ & Geographical origin & Host & Biovar & Race & AFLP cluster & Source $^{\mathrm{y}}$ \\
\hline POW30 & Yeongam, Jeollanam-do & Potato & 4 & 1 & II & This study \\
\hline POW33 & Yeongam, Jeollanam-do & Potato & 4 & 1 & IV & This study \\
\hline POW37 & Miryang, Gyeongsangnam-do & Potato & 4 & 1 & II & This study \\
\hline POW39 & Miryang, Gyeongsangnam-do & Potato & 4 & 1 & II & This study \\
\hline POW41 & Miryang, Gyeongsangnam-do & Potato & 4 & 1 & II & This study \\
\hline POW44 & Miryang, Gyeongsangnam-do & Potato & 4 & 1 & II & This study \\
\hline POW47 & Miryang, Gyeongsangnam-do & Potato & 4 & 1 & II & This study \\
\hline POW49 & Miryang, Gyeongsangnam-do & Potato & 4 & 1 & II & This study \\
\hline POW51 & Gimhae, Gyeongsangnam-do & Potato & 4 & 1 & IV & This study \\
\hline POW52 & Yeongam, Jeollanam-do & Potato & 4 & 1 & IV & This study \\
\hline POW53 & Yeongam, Jeollanam-do & Potato & 4 & 1 & IV & This study \\
\hline POW54 & Yeongam, Jeollanam-do & Potato & 4 & 1 & IV & This study \\
\hline POW55 & Yeongam, Jeollanam-do & Potato & 4 & 1 & IV & This study \\
\hline POW56 & Miryang, Gyeongsangnam-do & Potato & 4 & 1 & IV & This study \\
\hline POW57 & Wando, Jeollanam-do & Potato & 4 & 1 & IV & This study \\
\hline POW58 & Haenam, Jeollanam-do & Potato & 4 & 1 & IV & This study \\
\hline POW68 & Wando, Jeollanam-do & Potato & 4 & 1 & IV & This study \\
\hline POW69 & Wando, Jeollanam-do & Potato & 4 & 1 & IV & This study \\
\hline POW70 & Wando, Jeollanam-do & Potato & 4 & 1 & IV & This study \\
\hline POW71 & Wando, Jeollanam-do & Potato & 4 & 1 & IV & This study \\
\hline POW72 & Wando, Jeollanam-do & Potato & 4 & 1 & IV & This study \\
\hline POW73 & Wando, Jeollanam-do & Potato & 4 & 1 & IV & This study \\
\hline POW74 & Wando, Jeollanam-do & Potato & 4 & 1 & IV & This study \\
\hline POW75 & Wando, Jeollanam-do & Potato & 4 & 1 & IV & This study \\
\hline POW76 & Wando, Jeollanam-do & Potato & 4 & 1 & IV & This study \\
\hline TW14 & Chungju, Chungcheongbuk-do & Tomato & 4 & 1 & III & This study \\
\hline TW21 & Nonsan, Chungcheongnam-do & Tomato & 3 & 1 & III & This study \\
\hline TW23 & Pyeongtaek, Gyeonggi-do & Tomato & 4 & 1 & III & This study \\
\hline TW24 & Paju, Gyeonggi-do & Tomato & 4 & 1 & III & This study \\
\hline TW25 & Paju, Gyeonggi-do & Tomato & 4 & 1 & III & This study \\
\hline TW26 & Paju, Gyeonggi-do & Tomato & 4 & 1 & III & This study \\
\hline TW27 & Paju, Gyeonggi-do & Tomato & 4 & 1 & III & This study \\
\hline TW28 & Paju, Gyeonggi-do & Tomato & 4 & 1 & III & This study \\
\hline TW29 & Yeongcheon, Gyeongsangbuk-do & Tomato & 4 & 1 & III & This study \\
\hline TW30 & Yeongcheon, Gyeongsangbuk-do & Tomato & 4 & 1 & III & This study \\
\hline TW31 & Hadong, Gyeongsangnam-do & Tomato & 4 & 1 & I & This study \\
\hline TW32 & Hadong, Gyeongsangnam-do & Tomato & 4 & 1 & III & This study \\
\hline TW33 & Hadong, Gyeongsangnam-do & Tomato & 4 & 1 & III & This study \\
\hline TW38 & Gimje, Jeollabuk-do & Tomato & 4 & 1 & II & This study \\
\hline TW47 & Goseong, Gyeongsangnam-do & Tomato & 4 & 1 & II & This study \\
\hline TW56 & Gimhae, Gyeongsangnam-do & Tomato & 4 & 1 & II & This study \\
\hline TW60 & Gimcheon, Gyeongsangbuk-do & Tomato & 4 & 1 & II & This study \\
\hline TW64 & Gumi, Gyeongsangbuk-do & Tomato & 4 & 1 & II & This study \\
\hline TW65 & Gumi, Gyeongsangbuk-do & Tomato & 4 & 1 & II & This study \\
\hline TOW3 & Cheongwon, Chungcheongbuk-do & Tobacco & 3 & 1 & VI & This study \\
\hline PEW1 & Chilgok, Gyeongsangbuk-do & Peanut & 4 & 1 & $\mathrm{~N}^{\mathrm{z}}$ & This study \\
\hline SW1 & Pyeongtaek, Gyeonggi-do & Sunflower & 4 & 1 & $\mathrm{~N}$ & This study \\
\hline SEW2 & Yonchon, Gyeonggi-do & Sesame & 4 & 1 & $\mathrm{~N}$ & This study \\
\hline 7501 & Japan & Eggplant & 4 & 1 & II & Kyushu Unniv. \\
\hline 7502 & Japan & Eggplant & 4 & 1 & II & Kyushu Univ. \\
\hline 7601 & Japan & Eggplant & 4 & 1 & II & Kyushu Univ. \\
\hline N6211 & Japan & Pepper & 4 & 1 & II & Kyushu Univ. \\
\hline GMI1000 & Guyana & Tomato & 3 & 1 & $\mathrm{~N}$ & C. Boucher \\
\hline LMG2294 & Colombia & Potato & 2 & 3 & VII & BCCM/LMG \\
\hline LMG2296 & Zimbabwe & Tomato & 1 & 1 & $\mathrm{~N}$ & BCCM/LMG \\
\hline LMG2299 & United States & Tomato & 1 & 1 & $\mathrm{~N}$ & BCCM/LMG \\
\hline LMG2300 & Israel & Potato & 2 & 3 & VII & BCCM/LMG \\
\hline LMG2301 & Trinidad & Banana & 1 & 2 & $\mathrm{~N}$ & BCCM/LMG \\
\hline LMG2306 & Portugal & Tomato & 2 & 3 & VII & BCCM/LMG \\
\hline LMG17138 & Brazil & Potato & 1 & 1 & $\mathrm{~N}$ & BCCM/LMG \\
\hline LMG17139 & Cyprus & Potato & 2 & 3 & VII & BCCM/LMG \\
\hline LMG17140 & Sweden & Potato & 2 & 3 & VII & BCCM/LMG \\
\hline LMG17142 & U.K. & Potato & 2 & 3 & VII & $\mathrm{BCCM} / \mathrm{LMG}$ \\
\hline LMG17144 & Fiji & Potato & 3 & 1 & $\mathrm{~N}$ & BCCM/LMG \\
\hline
\end{tabular}


the test plants were immersed in $50 \mathrm{ml}$ of bacterial suspension for $2 \mathrm{~h}$ without artificial wounding. Control plant roots were immersed in sterile water. The inoculated plants (three tomato, three pepper, and three potato plants for each isolate) were planted in plastic pots and incubated in a greenhouse at 25 to $30^{\circ} \mathrm{C}$ under natural light. Symptom development was recorded daily for 15 days using the following scale: $-=$ no symptoms $;+=25 \%$ wilt symptoms, one or two leaves wilted; $++=50 \%$ wilt symptoms, three or four leaves wilted; and $+++=$ greater than $75 \%$ wilt symptoms, five leaves to whole plant wilted.

Biovar determination. Four hundred and seventy-eight isolates were classified into biovars based on their ability to oxidize three disaccharides (lactose, maltose, and cellobiose) and three hexose alcohols (mannitol, sorbitol, and dulcitol) as well as their ability to utilize trehalose, mesoinositol, and D-ribose. The characterization of the biovars was performed following the method of Hayward (9) with modifications. The bacterial cells were grown on TZC agar medium for $24 \mathrm{~h}$ at $28^{\circ} \mathrm{C}$ and then suspended in sterile distilled water $\left(10^{8} \mathrm{CFU} / \mathrm{ml}\right)$. Two $\mathrm{ml}$ of base medium (2 g $\mathrm{NH}_{4} \mathrm{H}_{2} \mathrm{PO}_{4}, 0.4 \mathrm{~g} \mathrm{KCl}, 0.4 \mathrm{~g} \mathrm{MgSO}_{4}$. $7 \mathrm{H}_{2} \mathrm{O}, 2$ g peptone, and $0.6 \mathrm{ml} 1 \%$ [wt/vol] bromothymol blue per liter, $\mathrm{pH}$ 7.1) containing $1 \%$ filter-sterilized lactose, maltose, cellobiose, mannitol, sorbitol, dulcitol, trehalose, meso-inositol, and Dribose were dispensed into the wells of 24-well tissue culture plates (SPL Life Sciences, Seoul, Korea). Each well was inoculated with $5 \mu \mathrm{l}$ of the prepared suspension, and the test was repeated three times with noninoculated controls. The plates were incubated at $28^{\circ} \mathrm{C}$ for 14 days, and color changes in each well were recorded daily.

AFLP analysis. Genomic DNA from 125 representative isolates (Table 1 ) representing different hosts, biovars, and geo- graphical origins was isolated as described by Sambrook et al. (24), followed by AFLP analysis as described by Poussier et al. (21) with modifications. Genomic DNA (100 ng/sample) was digested with $1 \mathrm{U}$ SacI (Takara Bio Inc., Otsu, Japan), 1 U MspI (Takara), and $2 \mu \mathrm{l} 10 \times$ OPA buffer $(500 \mathrm{mM}$ potassium acetate, $100 \mathrm{mM}$ magnesium acetate, $100 \mathrm{mM}$ Tris-acetate, $\mathrm{pH} 7.5$ ) at $25^{\circ} \mathrm{C}$ for $16 \mathrm{~h}$. Adapter ligation was achieved by adding 5 pmol SacI adapter (5'-TGTACGCAGTCTAC-3' and 5'-CTCGTAGACTGCGTACAAGCT-3'), 50 pmol MspI adapter (5'-GACGAT GAGTCCTGAA-3' and 5'-CGTTCAGGA CTCATC-3'), 35 U T4 DNA ligase (Takara), $2 \mu \mathrm{l}$ 10× T4 DNA ligase buffer (Takara), and sterilized water to make a final volume of $20 \mu \mathrm{l}$. The digested and ligated DNA was diluted 10-fold and stored at $-20^{\circ} \mathrm{C}$ until use. Selective amplifications were performed using two primers complementary to the adapter sequences with additional selective nucleotides at their $3^{\prime}$ ends (SacI-selective primer: 5'-CTCGTA GACTGCGTACAAGCTC-3', MspI-selective primer: 5'-GATGAGTCCTGAACG GCG-3'). Digested and ligated DNA (1.3 $\mu \mathrm{l})$ was added to $3.7 \mu \mathrm{l}$ of a mixture containing $0.5 \mu \mathrm{l} \mathrm{10 \times} \mathrm{AFLP} \mathrm{buffer}(500 \mathrm{mM}$ $\mathrm{KCl}, 100 \mathrm{mM}$ Tris-Cl [pH 7.0], $15 \mathrm{mM}$ $\mathrm{MgCl}_{2}$, and $1 \%$ Triton-X 100), $3.475 \mathrm{ng}$ SacI selective primer labeled with $\gamma$ $\left[{ }^{32} \mathrm{P}\right]$ ATP (Amersham Biosciences, Uppsala, Sweden), $7.54 \mathrm{ng}$ MspI selective primer, and $0.125 \mathrm{U} \mathrm{Taq}$ polymerase (Takara). DNA amplification by PCR was performed using a thermocycler (model PTC-200, MJ Research Inc., Waltham, MA) and the following program: 1 cycle of $30 \mathrm{~s}$ at $94^{\circ} \mathrm{C}, 30 \mathrm{~s}$ at $65^{\circ} \mathrm{C}, 1 \mathrm{~min}$ at $72^{\circ} \mathrm{C}$, and $30 \mathrm{~s}$ at $94^{\circ} \mathrm{C}$ followed by 13 cycles of 1 cycle of $30 \mathrm{~s}$ at $94^{\circ} \mathrm{C}, 23$ cycles of $30 \mathrm{~s}$ at $56^{\circ} \mathrm{C}$ (with a progressive decrease in annealing temperature of $0.7^{\circ} \mathrm{C}$ during each cycle), $1 \mathrm{~min}$ at $72^{\circ} \mathrm{C}, 30 \mathrm{~s}$ at $94^{\circ} \mathrm{C}$, and 1 cycle of $30 \mathrm{~s}$ at $56^{\circ} \mathrm{C}$ followed by $5 \mathrm{~min}$ at $72^{\circ} \mathrm{C}$. The amplified fragments were separated by electrophoresis on $6 \%$ polyacrylamide gels, and the patterns were visualized as described by Vos et al. (29). Individual DNA fragments, ranging between 150 and $1,000 \mathrm{bp}$, that were detected reproducibly were scored as present or absent. Binary matrices consisting of 0 (absence of a given band) and 1 (presence of a given band) were analyzed to obtain simple matching coefficients among the isolates using NTSYS-pc (version 2.0, Exeter Biological Software, Setauket, NY). The simple matching coefficients were then clustered to generate similarity trees with the SHAN clustering program, using the unweighted pair-group method with arithmetic average (UPGMA) algorithm in NYSYS-pc. Classification of the AFLP data was performed as described by Poussier et al. (21).

16S rRNA gene sequencing. The $16 \mathrm{~S}$ rRNA gene sequence was determined for 8 representative isolates (SL2249, SL2029, SL341, SL2025, TOW3, POW57, TW56, and N6211) taken from each AFLP cluster (I to VI). The 16S rRNA genes of the 8 isolates were amplified by PCR in $50-\mu \mathrm{l}$ reaction volumes containing $5 \mu \mathrm{l} 10 \times \mathrm{PCR}$ buffer (Takara), $5 \mu$ l each dNTP $(2.5 \mathrm{mM}$, Takara), $1 \mu \mathrm{l}$ each primer (100 pmol, 27mF: 5'-AGAGTTTGATCMTGGCTC AG-3', 1492mR: 5'-GGYTACCTTGTT ACGACTT-3'), $0.5 \mu \mathrm{l}$ of $\mathrm{Taq}$ polymerase (250 U/ $\mu \mathrm{l}$, Takara), and $2 \mu \mathrm{l}$ bacterial suspension $\left(A_{600 \mathrm{~nm}}=0.1\right)$. The amplifications were performed in an automated thermocycler (model PTC-200, MJ Research) with an initial denaturation at $94^{\circ} \mathrm{C}$ for 5 min, followed by 29 cycles of denaturation at $94^{\circ} \mathrm{C}$ for $1 \mathrm{~min}$, annealing at $55^{\circ} \mathrm{C}$ for 1 min, and extension at $72^{\circ} \mathrm{C}$ for $1.5 \mathrm{~min}$, with a final extension at $72^{\circ} \mathrm{C}$ for $10 \mathrm{~min}$. The amplified DNA was cloned into the SmaI site of pBluescript II $(\mathrm{KS}+)$ (Stratagene, Inc., La Jolla, CA) as described by Sambrook et al. (24). The se-

Table 2. The 16S rRNA nucleotide sequence differences among 18 isolates of Ralstonia solanacearum

\begin{tabular}{|c|c|c|c|c|c|c|c|c|c|c|c|c|c|c|c|c|c|c|c|c|c|c|c|c|c|c|}
\hline \multirow[b]{2}{*}{ Isolate $^{\mathrm{X}}$} & \multirow[b]{2}{*}{ Origin } & \multirow[b]{2}{*}{ Host } & \multirow[b]{2}{*}{$\begin{array}{l}\text { Bio- } \\
\text { var }\end{array}$} & \multirow{2}{*}{$\begin{array}{c}\text { Division } \\
\text { or sub- } \\
\text { divisiony }\end{array}$} & \multirow{2}{*}{$\begin{array}{c}\text { GenBank } \\
\text { accession } \\
\text { no. }\end{array}$} & \multicolumn{21}{|c|}{ Nucleotide(s) at position(s) ${ }^{\mathrm{z}}$} \\
\hline & & & & & & 152 & 165 & 167 & 169 & 269 & 375 & 387 & 397 & 410 & 416 & $\begin{array}{c}458- \\
460\end{array}$ & 474 & 649 & 669 & 737 & 923 & 1424 & 1428 & 1451 & 1456 & 1472 \\
\hline SL341 & Korea & Tomato & 3 & 1 & DQ924950 & $\mathrm{A}$ & $\mathrm{C}$ & A & $\mathrm{U}$ & $\mathrm{C}$ & $\mathrm{U}$ & $\mathrm{U}$ & $\mathrm{G}$ & G & $\mathrm{U}$ & $\mathrm{ACU}$ & $\mathrm{U}$ & G & $\mathrm{A}$ & $\mathrm{U}$ & $\mathrm{A}$ & $\mathrm{C}$ & $\mathrm{A}$ & $\mathrm{C}$ & A & $\mathrm{U}$ \\
\hline SL2025 & Korea & Sesame & 3 & 1 & DQ924951 & A & $\mathrm{C}$ & A & $\mathrm{C}$ & $\mathrm{C}$ & $\mathrm{U}$ & $\mathrm{U}$ & A & $\mathrm{G}$ & $\mathrm{G}$ & $\mathrm{ACU}$ & $\mathrm{U}$ & $\mathrm{G}$ & A & $\mathrm{U}$ & A & $\mathrm{C}$ & A & $\mathrm{C}$ & A & $\mathrm{U}$ \\
\hline SL2029 & Korea & Potato & 2 & $2 b$ & DQ924952 & A & $\mathrm{C}$ & G & $\mathrm{C}$ & $\mathrm{C}$ & $\mathrm{U}$ & $\mathrm{U}$ & A & G & G & UUC & $\mathrm{A}$ & $\mathrm{G}$ & G & $\mathrm{C}$ & A & $\mathrm{C}$ & A & $\mathrm{C}$ & A & $\mathrm{U}$ \\
\hline SL2249 & Korea & Potato & 1 & 1 & DQ924953 & A & $\mathrm{C}$ & A & $\mathrm{C}$ & $\mathrm{C}$ & $\mathrm{U}$ & $\mathrm{U}$ & A & G & $\mathrm{G}$ & $\mathrm{ACU}$ & $\mathrm{U}$ & $\mathrm{G}$ & A & $\mathrm{U}$ & A & $\mathrm{C}$ & A & $\mathrm{C}$ & A & $\mathrm{U}$ \\
\hline POW57 & Korea & Potato & 4 & 1 & DQ924954 & A & $\mathrm{C}$ & A & C & $\mathrm{C}$ & $\mathrm{U}$ & $\mathrm{C}$ & A & G & G & $\mathrm{ACU}$ & $\mathrm{U}$ & $\mathrm{G}$ & A & $\mathrm{U}$ & A & $\mathrm{C}$ & A & $\mathrm{C}$ & A & $\mathrm{U}$ \\
\hline TW56 & Korea & Tomato & 4 & 1 & DQ924957 & G & $\mathrm{C}$ & A & $\mathrm{C}$ & $\mathrm{C}$ & $\mathrm{U}$ & $\mathrm{U}$ & A & $\mathrm{A}$ & $\mathrm{G}$ & $\mathrm{ACU}$ & $\mathrm{U}$ & $\mathrm{G}$ & A & $\mathrm{U}$ & A & $\mathrm{C}$ & $\mathrm{A}$ & $\mathrm{C}$ & A & $\mathrm{U}$ \\
\hline TOW3 & Korea & Tobacco & 3 & 1 & DQ924955 & A & $\mathrm{C}$ & A & $\mathrm{C}$ & $\mathrm{C}$ & G & $\mathrm{U}$ & A & G & $\mathrm{G}$ & $\mathrm{ACU}$ & $U$ & $\mathrm{G}$ & A & $\mathrm{U}$ & A & $\mathrm{C}$ & $\mathrm{A}$ & $\mathrm{C}$ & $\mathrm{A}$ & $\mathrm{U}$ \\
\hline N6211 & Japan & Pepper & 4 & 1 & DQ924956 & A & $\mathrm{C}$ & A & $\mathrm{C}$ & $\mathrm{C}$ & $\mathrm{U}$ & $\mathrm{U}$ & A & G & $\mathrm{G}$ & $\mathrm{ACU}$ & $\mathrm{J}$ & $\mathrm{G}$ & A & $\mathrm{U}$ & G & $\mathrm{C}$ & A & $\mathrm{C}$ & A & $\mathrm{U}$ \\
\hline ACH0158 & Australia & Potato & 2 & $2 \mathrm{a}$ & U28224 & A & $\mathrm{C}$ & A & $\mathrm{C}$ & $\mathrm{C}$ & $\mathrm{U}$ & $\mathrm{U}$ & A & G & G & UUC & $\mathrm{A}$ & A & A & $\mathrm{U}$ & A & $\mathrm{U}$ & G & $\mathrm{C}$ & G & $\mathrm{C}$ \\
\hline ACH0171 & Australia & Eggplant & 3 & 1 & U27986 & A & C & A & C & $\mathrm{C}$ & $\mathrm{U}$ & $\mathrm{U}$ & A & G & G & $\mathrm{ACU}$ & $\mathrm{I} U$ & G & A & $\mathrm{U}$ & A & C & A & C & A & $\mathrm{U}$ \\
\hline CFBP712 & Burkina Faso & Eggplant & 1 & $2 \mathrm{a}$ & AF207892 & A & $\mathrm{C}$ & A & $\mathrm{C}$ & $\mathrm{U}$ & $\mathrm{U}$ & $\mathrm{U}$ & A & G & G & UUC & $A$ & G & A & $\mathrm{U}$ & A & $\mathrm{U}$ & G & $\mathrm{U}$ & G & $\mathrm{C}$ \\
\hline CFBP734 & Madagascar & Potato & 1 & $2 c$ & AF207895 & A & $\mathrm{U}$ & A & $\mathrm{C}$ & $\mathrm{C}$ & $\mathrm{U}$ & $\mathrm{U}$ & A & G & G & UCU & $\mathrm{A}$ & $\mathrm{G}$ & A & $\mathrm{U}$ & A & $\mathrm{U}$ & G & $\mathrm{U}$ & G & $\mathrm{C}$ \\
\hline GMI1000 & Guyana & Tomato & 3 & 1 & AL646052 & A & $\mathrm{C}$ & A & $\mathrm{C}$ & $\mathrm{C}$ & $\mathrm{U}$ & $\mathrm{U}$ & A & $\mathrm{G}$ & $\mathrm{G}$ & $\mathrm{ACU}$ & $\mathrm{U}$ & $\mathrm{G}$ & A & $\mathrm{U}$ & A & $\mathrm{C}$ & $\mathrm{A}$ & $\mathrm{C}$ & A & $\mathrm{U}$ \\
\hline MAFF211266 & Japan & Tomato & 4 & 1 & AF207897 & A & $\mathrm{C}$ & A & $\mathrm{C}$ & $\mathrm{C}$ & $\mathrm{U}$ & $\mathrm{U}$ & A & $\mathrm{G}$ & $\mathrm{G}$ & $\mathrm{ACU}$ & $\mathrm{U}$ & $\mathrm{G}$ & A & $\mathrm{U}$ & A & $\mathrm{C}$ & $\mathrm{A}$ & $\mathrm{C}$ & A & $\mathrm{U}$ \\
\hline MAFF301559 & Japan & Potato & N2 & $2 b$ & AB024608 & A & $\mathrm{C}$ & G & $\mathrm{C}$ & $\mathrm{C}$ & $\mathrm{U}$ & $\mathrm{U}$ & $\mathrm{A}$ & $\mathrm{G}$ & $\mathrm{G}$ & UUC & $\mathrm{A}$ & $\mathrm{G}$ & G & $\mathrm{C}$ & A & $\mathrm{C}$ & A & $\mathrm{C}$ & A & $\mathrm{U}$ \\
\hline NCPPB 1018 & Angola & Potato & 1 & $2 c$ & AF207893 & A & $\mathrm{U}$ & $\mathrm{A}$ & $\mathrm{C}$ & $\mathrm{C}$ & $\mathrm{U}$ & $\mathrm{U}$ & A & $\mathrm{G}$ & G & $\mathrm{UCU}$ & $\mathrm{A}$ & G & A & $\mathrm{U}$ & A & $\mathrm{U}$ & G & $\mathrm{U}$ & G & $\mathrm{C}$ \\
\hline R142 & Indonesia & Clove & 2 & $2 b$ & U28233 & A & $\mathrm{C}$ & G & $\mathrm{C}$ & $\mathrm{C}$ & $\mathrm{U}$ & $\mathrm{U}$ & A & G & G & UUC & $\mathrm{A}$ & G & G & $\mathrm{C}$ & A & $\mathrm{C}$ & A & $\mathrm{C}$ & A & $\mathrm{U}$ \\
\hline R791 & Indonesia & Tomato & 3 & 1 & U27987 & A & $\mathrm{C}$ & A & $\mathrm{C}$ & $\mathrm{C}$ & $\mathrm{U}$ & $\mathrm{U}$ & A & G & $\mathrm{G}$ & $\mathrm{ACU}$ & $\mathrm{U}$ & G & A & $\mathrm{U}$ & A & $\mathrm{C}$ & A & $\mathrm{C}$ & A & $\mathrm{U}$ \\
\hline
\end{tabular}

\footnotetext{
$\mathrm{x}$ Except for the first 8 isolates, data shown are from Horita and Tsuchiya (12), Poussier et al. (21), and Taghavi et al. (27).

y Based on data from Poussier et al. (21) and Taghavi et al. (27).
}

z Escherichia coli numbering (30). 
quences of both strands of the 16S rRNA gene from each isolate were determined using an ABI3730XL automated DNA sequencer (Applied Biosystems Inc., Foster City, CA), and the sequences were aligned using the Clustal X program (14) with the published $(12,21,27)$ 16S rRNA gene sequences for the following $R$. solanacearum isolates: ACH0158, ACH0171, CFBP712, CFBP734, GMI1000, MAFF211266, MAFF301559, NCPPB1018, R142, and R791. Sequence differences and their positions (using the Escherichia coli numbering system [30]) are shown in Table 2. A dendrogram was constructed using the UPGMA method of the Molecular Evolutionary Genetics Analysis (MEGA 3.1) program (18). The strength of the tree branches was tested using 1,000 bootstrap trials. Classification of the sequences was performed as described by Poussier et al. (21) and Taghavi et al. (27).

Phylotype analysis. The endoglucanase, $h r p B$, and mutS gene sequences of the 8 representative isolates (SL2249, SL2029, SL341, SL2025, TOW3, POW57, TW56, and N6211) used for the 16S rRNA gene sequence analysis were determined for phylotype analysis. The endoglucanase and $h r p B$ genes were amplified as described by Poussier et al. (20) with modifications, and the mutS gene was amplified as described by Prior and Fegan (23) with modifications.

The endoglucanase gene of the 8 isolates was amplified by PCR in $50-\mu 1$ reaction volumes containing $5 \mu \mathrm{l} 10 \times P f u$ polymerase buffer (Stratagene), $5 \mu$ of each $2.5 \mathrm{mM}$ dNTP, $1 \mu \mathrm{l}$ of each primer (100 pmol, Endo-F: 5'-ATGCATGCCGCTGGT CGCCGC-3', Endo-R: 5'-GCGTTGCCC
GGCACGAACACC-3'), $1.25 \mathrm{U}$ of $P f u$ Turbo DNA Polymerase (Stratagene), and $2 \mu \mathrm{l}$ of bacterial suspension $\left(A_{600 \mathrm{~nm}}=0.1\right)$. The amplifications were performed in an automated thermocycler (model PTC-200, MJ Research) with an initial denaturation at $96^{\circ} \mathrm{C}$ for $5 \mathrm{~min}$, followed by 30 cycles of denaturation at $95^{\circ} \mathrm{C}$ for $1 \mathrm{~min}$, annealing at $70^{\circ} \mathrm{C}$ for $1 \mathrm{~min}$, and extension at $72^{\circ} \mathrm{C}$ for $2 \mathrm{~min}$, with a final extension at $72^{\circ} \mathrm{C}$ for $10 \mathrm{~min}$.

The $h r p B$ gene of the 8 isolates was amplified by PCR in $50-\mu l$ reaction volumes containing $5 \mu \mathrm{l}$ of $10 \times P f u$ polymerase buffer (Stratagene), $5 \mu \mathrm{l}$ of each $2.5 \mathrm{mM}$
dNTP, $1 \mu$ l of each primer (100 pmol, RShrpBf: 5'-TGCCATGCTGGGAAACAT CT-3', RShrpBr: 5'-GGGGGCTTCGTT GAACTGC-3'), $1.25 \mathrm{U}$ of $P f u$ Turbo DNA Polymerase (Stratagene), and $2 \mu \mathrm{l}$ bacterial suspension $\left(A_{600 \mathrm{~nm}}=0.1\right)$. The amplifications were performed in an automated thermocycler (model PTC-200, MJ Research) with an initial denaturation at $95^{\circ} \mathrm{C}$ for $5 \mathrm{~min}$, followed by 10 cycles of denaturation at $95^{\circ} \mathrm{C}$ for $30 \mathrm{~s}$, annealing at $64^{\circ} \mathrm{C}$ for $30 \mathrm{~s}$, and extension at $68^{\circ} \mathrm{C}$ for $2 \mathrm{~min}$. The final 20 cycles were the same as the first 10 except that an additional $20 \mathrm{~s}$ were added to the elongation step for each new

Table 4. Pathogenicity of Korean Ralstonia solanacearum isolates

\begin{tabular}{|c|c|c|c|c|c|c|}
\hline \multirow[b]{2}{*}{ Race } & \multirow{2}{*}{$\begin{array}{l}\text { Biovar (no. } \\
\text { of isolates) }\end{array}$} & \multirow[b]{2}{*}{ Host } & \multicolumn{3}{|c|}{ Pathogenicity $^{y}$} & \multirow{2}{*}{$\begin{array}{l}\text { AFLPz } \\
\text { cluster }\end{array}$} \\
\hline & & & Tomato & Pepper & Potato & \\
\hline 1 & $\begin{array}{l}1(3) \\
4(9)\end{array}$ & $\begin{array}{l}\text { Paprika } \\
\text { Potato } \\
\text { Tomato }\end{array}$ & +++ & + & +++ & I, VI \\
\hline 1 & $\begin{array}{l}3(48) \\
4(356)\end{array}$ & $\begin{array}{l}\text { Pepper } \\
\text { Potato } \\
\text { Sesame } \\
\text { Tobacco } \\
\text { Tomato }\end{array}$ & +++ & +++ & +++ & $\begin{array}{l}\text { I, II, } \\
\text { III, IV }\end{array}$ \\
\hline 1 & $4(16)$ & $\begin{array}{l}\text { Eggplant } \\
\text { Potato } \\
\text { Tomato }\end{array}$ & +++ & - & +++ & II, III \\
\hline 1 & $3(5)$ & Tomato & +++ & +++ & - & III \\
\hline 1 & $3(3)$ & Tobacco & +++ & - & + & VI \\
\hline 3 & $2(38)$ & $\begin{array}{l}\text { Potato } \\
\text { Tomato }\end{array}$ & + & - & +++ & $\mathrm{V}$ \\
\hline
\end{tabular}

y Development of symptoms was recorded daily for 15 days according to the following scale: $-=$ no symptoms; $+=25 \%$ wilt symptoms, one or two leaves wilted; $++=50 \%$ wilt symptoms, three or four leaves wilted; and +++ = greater than $75 \%$ wilt symptoms, five leaves to whole plant wilted. The pathogenicity test was repeated three times with three replications.

z Amplified fragment length polymorphism.

Table 3. Ralstonia solanacearum isolates used in endoglucanase, $h r p B$, and $m u t S$ gene sequence analyses

\begin{tabular}{|c|c|c|c|c|c|c|c|}
\hline \multirow[b]{2}{*}{ Isolate $^{x}$} & \multirow[b]{2}{*}{ Origin } & \multirow[b]{2}{*}{ Host } & \multirow[b]{2}{*}{ Biovar } & \multirow[b]{2}{*}{ Phylotype ${ }^{y}$} & \multicolumn{3}{|c|}{ GenBank accession no. } \\
\hline & & & & & $\begin{array}{l}\text { Endoglucanase } \\
\text { gene sequence }\end{array}$ & $\begin{array}{l}\text { hrpB gene } \\
\text { sequence }\end{array}$ & $\begin{array}{l}\text { mutS gene } \\
\text { sequence }\end{array}$ \\
\hline SL341 & Korea & Tomato & 3 & I & EF523197 & EF523205 & EF523213 \\
\hline SL2025 & Korea & Sesame & 3 & I & EF523199 & EF523207 & EF523215 \\
\hline SL2029 & Korea & Potato & 2 & IV & EF523198 & EF523206 & EF523214 \\
\hline SL2249 & Korea & Potato & 1 & I & EF523200 & EF523208 & EF523216 \\
\hline POW57 & Korea & Potato & 4 & I & EF523202 & EF523210 & EF523218 \\
\hline TW56 & Korea & Tomato & 4 & I & EF523201 & EF523209 & EF523217 \\
\hline TOW3 & Korea & Tobacco & 3 & I & EF523203 & EF523211 & EF523219 \\
\hline N6211 & Japan & Pepper & 4 & I & EF523204 & EF523212 & EF523220 \\
\hline CFBP712 & Burkina Faso & Eggplant & 1 & II-bhr & AF295267 & AF295620 & AY756773 \\
\hline CFBP734 & Madagascar & Potato & 1 & III & AF295274 & AF295627 & AY756746 \\
\hline CFBP2972 & Martinique & Potato & 1 & II-bhr & AF295264 & AF295617 & AY756807 \\
\hline CFBP3858 & Netherlands & Potato & 2 & II-nhr & AF295259 & AF295612 & AY756748 \\
\hline GMI1000 & Guyana & Tomato & 3 & I & AF295251 & AF295604 & AY756804 \\
\hline JT516 & Reunion Island & Potato & 2 & II-nhr & AF295258 & AF295611 & AY756783 \\
\hline MAFF211266 & Japan & Tomato & 4 & I & AF295250 & AF295603 & AY756791 \\
\hline MAFF301558 & Japan & Potato & $\mathrm{N} 2$ & IV & AY465002 & AY465037 & AY756812 \\
\hline MAFF301559 & Japan & Potato & N2 & IV & AY465001 & AY465036 & $\mathrm{N}^{\mathrm{z}}$ \\
\hline NCPPB1018 & Angola & Potato & 1 & III & AF295271 & AF295624 & AY756772 \\
\hline NCPPB3190 & Malaysia & Tomato & 3 & I & AF295253 & AF295606 & AY756738 \\
\hline R142 & Indonesia & Clove & 2 & IV & AY465003 & AY465038 & $\mathrm{N}$ \\
\hline UW9 & Costa Rica & Heliconia sp. & 1 & II-nhr & AF295257 & AF295610 & AY756744 \\
\hline UW162 & Peru & Musa sp. & 1 & II-nhr & AF295256 & AF295609 & AY756795 \\
\hline
\end{tabular}

${ }^{\mathrm{x}}$ Except for the first 8 isolates, the data shown are from Poussier et al. (20), Prior and Fegan (23), and Villa et al. (28).

${ }^{y}$ Based on data from Fegan and Prior (7) and Prior and Fegan (23). bhr and nhr denote broad host range and narrow host range, respectively.

${ }^{\mathrm{z}} \mathrm{N}$ : no available sequence. 
cycle, and a final extension at $68^{\circ} \mathrm{C}$ for 7 min was added.

The $m u t S$ gene of the 8 isolates was amplified by PCR in $50-\mu l$ reaction volumes containing $5 \mu \mathrm{l}$ of $10 \times P f u$ polymerase buffer (Stratagene), $5 \mu \mathrm{l}$ of each $2.5 \mathrm{mM}$ dNTP, $1 \mu$ of each primer $(100$ pmol, mutS-RsF.1570: 5'-ACAGCGCCTTGAGCC GGTACA-3', mutS-RsR.1926: 5'-GCT GATCACCGGCCCGAACAT-3'), $1.25 \mathrm{U}$ of $P f u$ Turbo DNA Polymerase (Stratagene), and $2 \mu \mathrm{l}$ bacterial suspension $\left(A_{600 \mathrm{~nm}}\right.$ $=0.1$ ). The amplifications were performed in an automated thermocycler (model
PTC-200, MJ Research) with an initial denaturation at $96^{\circ} \mathrm{C}$ for $5 \mathrm{~min}$, followed by 35 cycles of denaturation at $94^{\circ} \mathrm{C}$ for 1 min, annealing at $66^{\circ} \mathrm{C}$ for $1 \mathrm{~min}$, and extension at $72^{\circ} \mathrm{C}$ for $1.5 \mathrm{~min}$, with a final extension at $72^{\circ} \mathrm{C}$ for $5 \mathrm{~min}$.

The amplified DNA fragments were separated by $1.5 \%$ agarose gel electrophoresis in $0.5 \times \mathrm{TBE}$ buffer, purified using the MEGAquick-spin PCR \& Agarose gel DNA extraction system (Intron Biotechnology, Seoul, Korea) according to the manufacturer's instructions, and sequenced on both strands. The primers used to se-

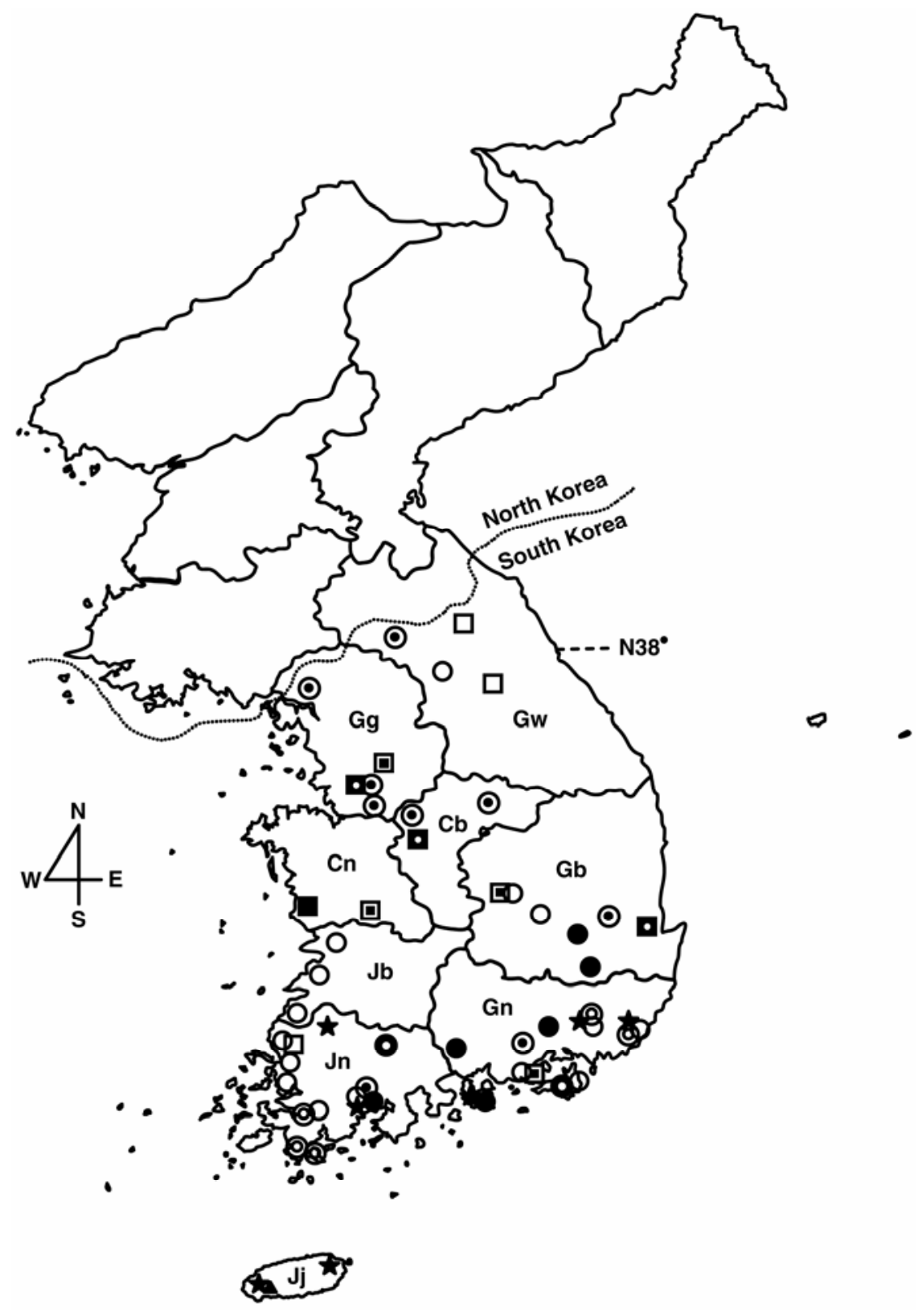

Fig. 1. Locations in Korea where Ralstonia solanacearum isolates were collected (Gg: Gyeonggi-do; Gw: Gangwon-do; Cb: Chungcheongbuk-do; Cn: Chungcheongnam-do; Jb: Jeollabuk-do; Jn: Jeollanam-do; Gb: Gyeongsangbuk-do; Gn: Gyeongsangnam-do; Jj: Jeju-do). Symbols indicate isolates ( $\mathbf{\Delta}$ : biovar 1, race 1, amplified fragment length polymorphism (AFLP) cluster I; $\mathbf{\square}$ : biovar 3, race 1, AFLP cluster I; $\square$ : biovar 3, race 1, AFLP cluster II; $\square$ : biovar 3, race 1, AFLP cluster III; $\mathbf{0}$ : biovar 3, race 1 , AFLP cluster VI; 0 : biovar 4 , race 1 , AFLP cluster I; $\bigcirc$ : biovar 4, race 1, AFLP cluster II; $\odot:$ biovar 4, race 1, AFLP cluster III; ๑: biovar 4, race 1, AFLP cluster IV; 0: biovar 4, race 1, AFLP cluster VI; $\star$ : biovar 2, race 3 , AFLP cluster V). Each symbol indicates representative locations for the same geographical origin, host, biovar, race, and AFLP cluster. quence endoglucanase, $h r p B$, and mutS were: Endo-F/Endo-R for the endoglucanase gene, RShrpBf/RShrpBr for $h r p B$, and mutS-RsF.1570/mutS-RsR.1926 for mutS $(20,23)$. The partial sequences were aligned using the Clustal $\mathrm{X}$ program (14) with the published $(20,23,28)$ endoglucanase, $h r p B$, and mutS gene sequences for the following $R$. solanacearum isolates: CFBP712, CFBP734, CFBP2972, CFBP3858, GMI1000, JT516, MAFF211266, MAFF301558, MAFF301559, NCPPB1018, NCPPB3190, R142, UW9, and UW162. The partial sequences obtained in this study have been deposited in the GenBank database under the accession numbers listed in Table 3. Evolutionary distances between the sequences were computed using the algorithm of Jukes and Cantor (16), and phylogenetic trees were constructed from the genetic distance data using the neighbor-joining method in the Molecular Evolutionary Genetics Analysis (MEGA 3.1) program (18). The strength of the tree branches was tested using 1,000 bootstrap trials. Classification of the sequences was performed as described by Fegan and Prior (7) and Prior and Fegan (23).

\section{RESULTS}

Host range and biovar determination. The Korean isolates were tested for biovar determination and pathogenicity on various host plants. Of the 478 isolates tested, 3 were biovar 1, 38 were biovar 2, 56 were biovar 3, and 381 were biovar 4 (Table 4). Among the Korean biovar 2 isolates, none were identified as biovar N2. All biovar 1 isolates were pathogenic on tomato and potato and weakly pathogenic on pepper (Table 4). Among the 56 biovar 3 isolates, 48 were pathogenic on tomato, pepper, and potato, 5 were pathogenic on tomato and pepper but nonpathogenic on potato, and 3 were pathogenic on tomato and weakly pathogenic on potato but nonpathogenic on pepper (Table 4). Of the 381 biovar 4 isolates, 356 were pathogenic on tomato, pepper, and potato, 16 were pathogenic on tomato and potato and nonpathogenic on pepper, and 9 were pathogenic on tomato and potato and weakly pathogenic on pepper (Table 4). Overall, all biovar 1, 3, and 4 isolates belonged to race 1 , and all biovar 2 isolates from tomato and potato were pathogenic on potato and weakly pathogenic or nonpathogenic on tomato and pepper, corresponding to race 3 (Table 4). These results indicate that $92 \%$ (440 of 478 isolates) of the Korean isolates belong to race 1 , and $85 \%$ (404 of 478 isolates) of them are highly virulent on tomato, pepper, and potato.

Geographical distribution. Figure 1 shows the geographical distribution of the various Korean isolates, reflecting their genetic diversity. Biovar 1 isolates of race 1 taken from tomato and potato plants were present only in Jeju-do (Fig. 1). Bio- 
var 2 isolates belonging to race 3 were found at 2 locations in Jeju-do, 2 locations in Jeollanam-do, and 3 locations in Gyeongsangnam-do (Fig. 1). Biovar 3 isolates belonging to race 1 were collected from wilted tomato, potato, tobacco, eggplant, pepper, sesame, and paprika plants at 2 locations in Gangwon-do, 4 locations in Gyeonggi-do, 4 locations in Chungcheongbuk-do, 6 locations in Chungcheongnam-do, 3 locations in Jeollanamdo, 4 locations in Gyeongsangbuk-do, 4 locations in Gyeongsangnam-do, and 2 locations in Jeju-do (Fig. 1). Biovar 4 isolates belonging to race 1 were the most widespread among the four biovars. These isolates were recovered from diseased tomato, potato, pepper, eggplant, sesame, paprika, pimiento, peanut, and sunflower plants at 6 locations in Gangwon-do, 10 locations in Gyeonggi-do, 7 locations in Chungcheongbuk-do, 4 locations in Chungcheongnam-do, 3 locations in Jeollabuk-do, 9 locations in Jeollanam-do, 10 locations in Gyeongsangbuk-do, 11 locations in Gyeongsangnam-do, and 2 locations in Jeju-do (Fig. 1).

AFLP analysis. A total of 110 DNA fragments ranging from 150 to $1,000 \mathrm{bp}$, which were consistently produced, were selected to analyze 109 Korean and 16 foreign isolates. Among the isolates tested, 62 unique AFLP fragments were identified, and among those 10 were present only in the Korean biovar 2 (race 3) isolates (Fig. 2). A dendrogram generated by NTSYS-pc separated the isolates into two divisions: the first contained all 109 Korean isolates, 4 Japanese isolates, and the Guyanese isolate (GMI1000), while the second contained the 11 LMG isolates (Fig. 3). Isolates belonging to the first division were further divided into six clusters, designated I through VI, at a 0.62 correlation level. Three Korean isolates (SW1, SEW2, and PEW1) and GMI1000 did not fit into any of the clusters (Fig. 3) at the 0.62 correlation level. The first cluster, with a coefficient of 0.75 , contained biovar 1,3 , and 4 (race 1) isolates from tomato, pepper, potato, and sesame collected in Chungcheongnam-do, Gyeongsangnam-do, Gyeongsangbuk-do, and Jeollanam-do (Figs. 1 and 3). The second cluster, with a coefficient of 0.70 , contained biovar 3 and 4 (race 1) isolates collected from tomato, pepper, potato, and eggplant in Gyeongsangbuk-do, Gyeongsangnam-do, Jeollabuk-do, and Jeollanam-do as well as biovar 4 isolates from eggplant and pepper in Japan (Figs. 1 and 3). The third cluster, with a coefficient of 0.72 , included biovar 3 and 4 (race 1) isolates collected from tomato plants in Gangwon-do, Gyeonggido, Chungcheongbuk-do, Chungcheongnam-do, Gyeongsangbuk-do, Gyeongsangnam-do, and Jeollanam-do (Figs. 1 and 3). The fourth cluster, with a coefficient of 0.70 , included biovar 4 (race 1 ) isolates from potato collected in Jeol- lanam-do and Gyeongsangnam-do (Figs. 1 and 3 ). The fifth cluster, with a coefficient of 0.62 , included biovar 2 (race 3 ) isolates from tomato and potato collected in Jeollanam-do, Gyeongsangnam-do, and Jejudo (Figs. 1 and 3). The sixth cluster, with a coefficient of 0.685 , included biovar 3 and 4 (race 1) isolates from paprika and tobacco collected in Gyeonggi-do, Chungcheongbuk-do, Gyeongsangbuk-do, and Gyeongsangnam-do (Figs. 1 and 3). The Korean isolates and isolate GMI1000 had a genetic similarity coefficient of 0.59 . The second division, with a coefficient of 0.48 , included cluster contained biovar 2 isolates originating from 11 countries (Fig. 3, Table 1).

16S rRNA gene sequence analysis. We sequenced the complete 16S rRNA genes from 7 Korean isolates (biovar 1: SL2249; biovar 1 and 3 isolates, and the seventh

biovar 2: SL2029; biovar 3: SL341, SL2025, and TOW3; and biovar 4: POW57 and TW56) and 1 Japanese isolate (biovar 4: N6211). By comparing 1,468 nucleotides, all of the isolates were grouped into four clusters (Fig. 4A). Among them, the Korean and Japanese isolates were separated into two groups. Cluster I contained isolates of biovars 1,3 , and 4 (race 1), and cluster II contained a biovar 2 (race 3 ) isolate (Fig. 4A). The sequences of the cluster I isolates were identical, while the cluster II isolates differed from the cluster I isolates at seven nucleotide positions. Comparative analysis of the 16S rRNA gene sequences from the 7 Korean isolates, 1 Japanese isolate, and 10 other foreign isolates was performed based on previously published work by Horita and Tsuchiya (12), Poussier et al. (21), and

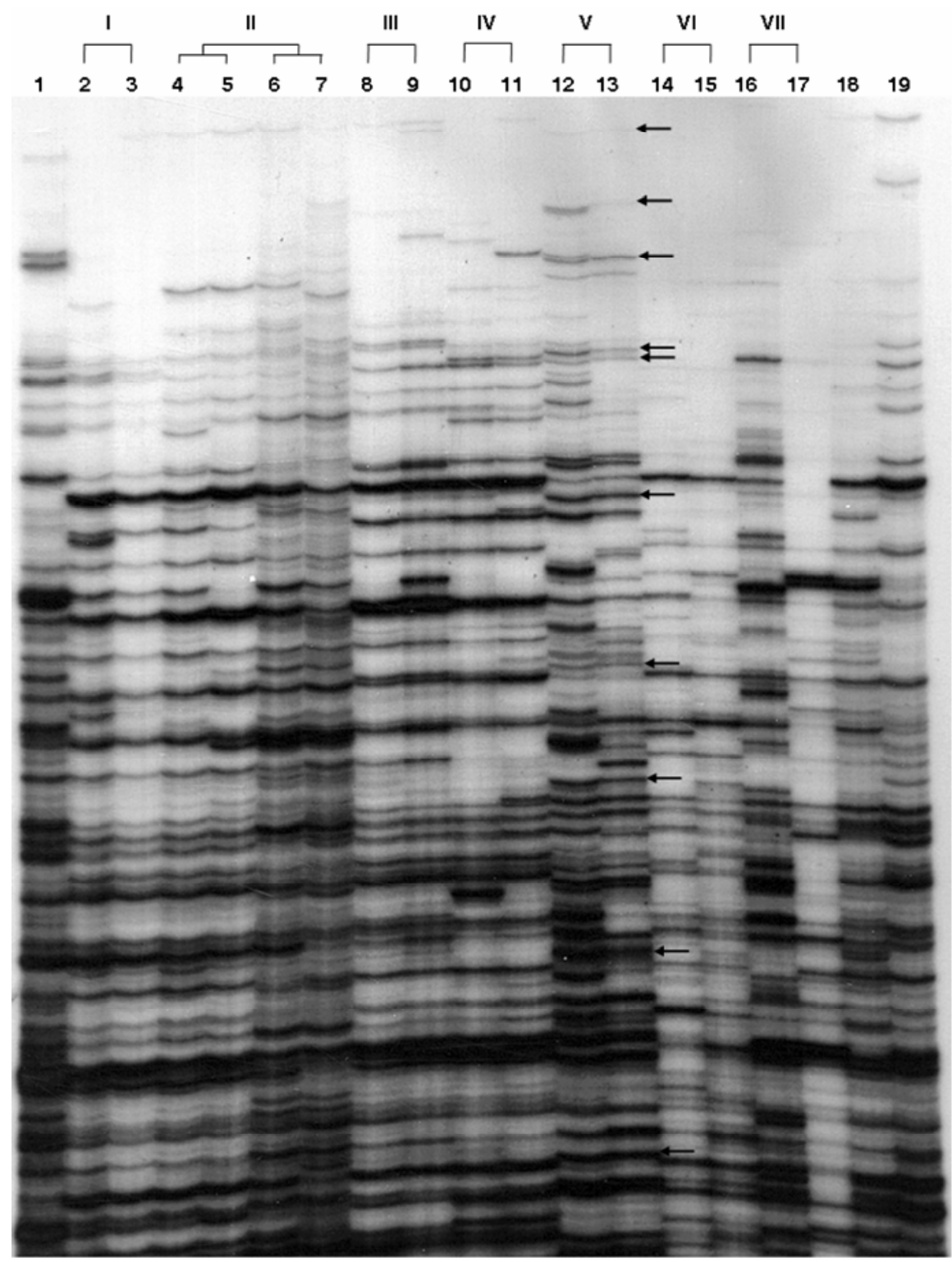

Fig. 2. Amplified fragment length polymorphism (AFLP) fingerprints of Ralstonia solanacearum isolates. Lanes: 1, GMI1000; 2, PW17; 3, PW33; 4, POW1; 5, POW7; 6, N6211; 7, 7601; 8, SL341; 9 TW30; 10, POW55; 11, POW57; 12, SL2029; 13, SL2313; 14, SL1977; 15, TOW3; 16, LMGG2296; 17, LMG17138; 18, PEW1; 19, SEW2. Arrows indicate specific fragments in the cluster V (biovar 2) isolates. 
Division Cluster Biovar Race

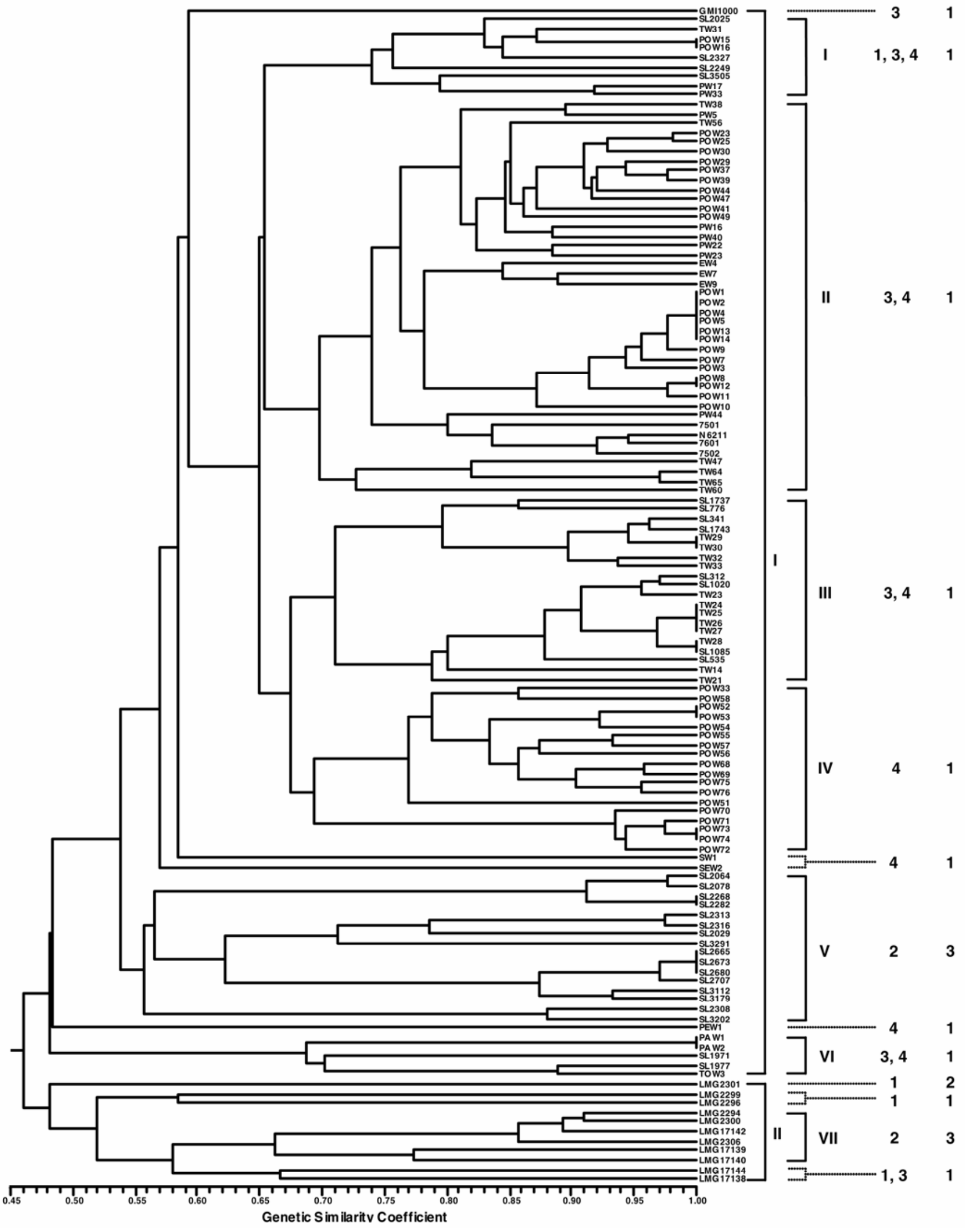

Fig. 3. Dendrogram constructed by the unweighted pair-group method with the arithmetic average showing the correlation between amplified fragment length polymorphism (AFLP) fingerprints of the Ralstonia solanacearum isolates. Roman numerals indicate clusters. LMG: Laboratorium voor Microbiologie Universiteit Gent, Belgium. 
Taghavi et al. (27). Taghavi et al. (27) reported that nucleotides $458,459,460$, and 474 (E. coli numbering) are unique and conserved between the divisions; therefore, we compared those positions as well as others that we previously reported to be variable. The cluster I isolates (race 1), which belong to division 1 according to Taghavi et al. (27), had the same nucleotides at positions 458 to 460 (ACU) and 474 (U), as did those of the Japanese isolate (biovar 4: MAFF211266), an Australian isolate (biovar 3: ACH0171), an Indonesian isolate (biovar 3: R791), and the Guyanese isolate (biovar 3: GMI1000) (Table 2, Fig. 4A). Six of the 7 Korean isolates belonged to cluster I. The cluster II isolates, which belong to subdivision $2 b$, including Korean biovar 2 isolate SL2029, Indonesian biovar 2 isolate R142, and Japanese biovar N2 isolate MAFF301559, had identical sequences (Table 2, Fig. 4A). The cluster III isolates, including African biovar 1 isolate CFBP712 and Australian biovar 2 isolate $\mathrm{ACH} 0158$, were distinct from the other isolates at nucleotides 458 to 460 (UUC), 474 (A), 1424 (U), 1428 (G), $1456(\mathrm{G})$, and $1472(\mathrm{C})$, but were identical at positions 458 to 460,474 , $1424,1428,1456$, and 1472. Therefore, these isolates belong to subdivision $2 \mathrm{a}$ according to Taghavi et al. (27) (Table 2, Fig. 4A). The cluster IV isolates, including the African biovar 1 isolates CFBP734 and NCPPB1018, differed from the other isolates by having a $U$ at position 165 and UUC at 458 to 460 (Table 2); consequently, these isolates were grouped in subdivision $2 \mathrm{c}$ according to the classification of Poussier et al. (21) (Table 2, Fig. $4 \mathrm{~A})$. The bootstrap value for the $16 \mathrm{~S}$ rRNA gene sequences among the cluster I isolates ranged from 19 to $84 \%$, while the bootstrap value among cluster II was $99 \%$. The bootstrap values for clusters III and IV were 61 and $98 \%$, respectively, and between clusters III and IV was $83 \%$ (Fig. 4A).
Phylogenetic analysis. Partial endoglucanase gene sequences (694 nucleotides) covering approximately $45 \%$ of the complete endoglucanase gene from the 8 isolates used for $16 \mathrm{~S}$ rRNA gene sequence analysis and 14 reference isolates were compared and divided into four clusters (Table 3, Fig. 4B). Among them, the 7 Korean isolates and one Japanese isolate belonged to either cluster I or cluster II (Fig. 4B). The cluster I isolates belonged to phylotype I along with the Japanese isolate (MAFF211266), the Malaysian isolate (NCPPB3190), and the Guyanese isolate (GMI1000; Fig. 4B). The cluster II isolates, composed of Korean biovar 2 isolate SL2029, Indonesian biovar 2 isolate R142, and Japanese biovar N2 isolates MARR301558 and MAFF301559, belonged to phylotype IV (Table 3, Fig. 4B). The cluster III isolates, including the biovar 1 African isolates NCPPB1018 and CFBP734, corresponded to phylotype III (Table 3, Fig. 4B). The cluster IV isolates
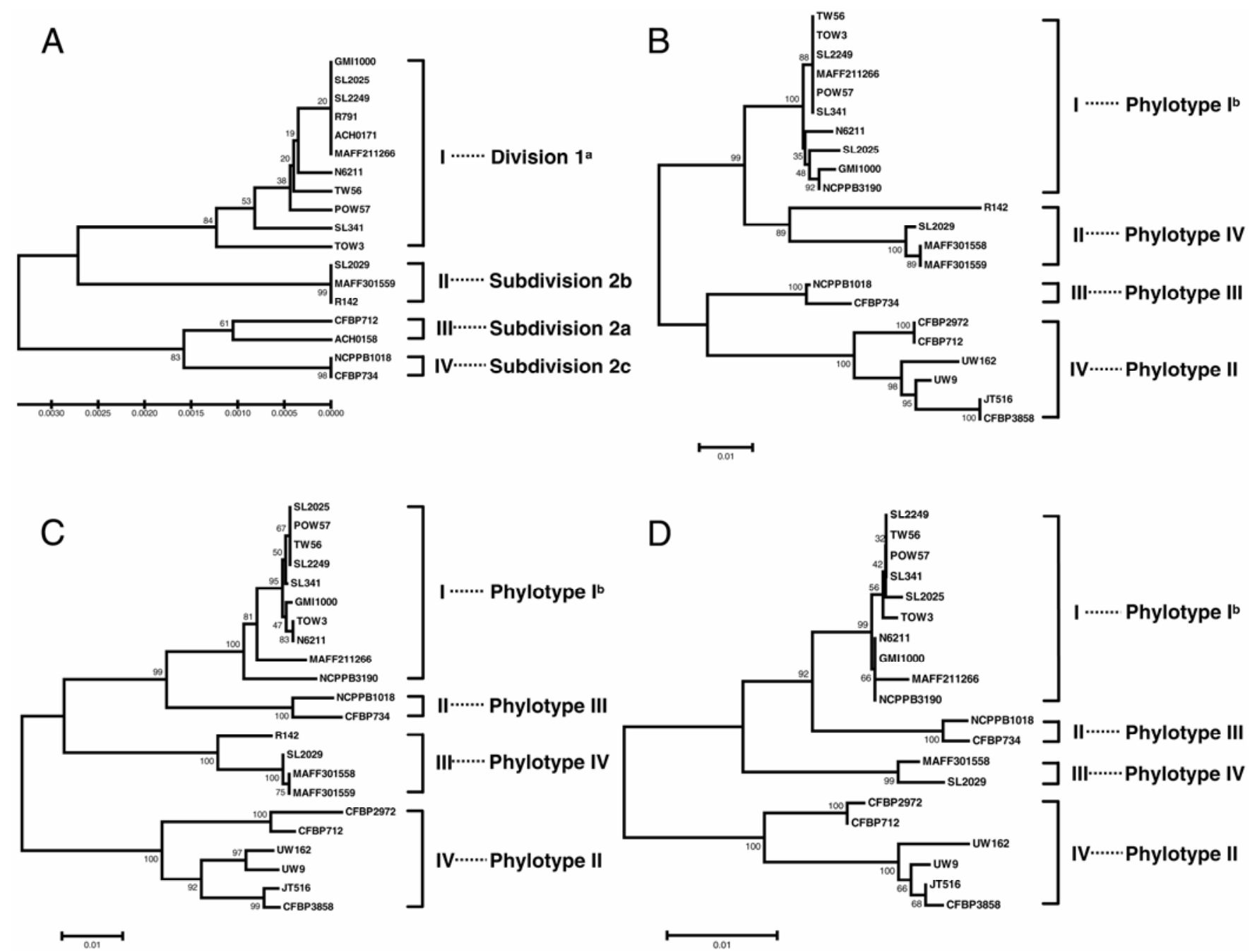

Fig. 4. A, Dendrogram constructed by the unweighted pair-group method with the arithmetic average showing the phylogenetic relationships among Ralstonia solanacearum strains based on a comparison of their 16S rRNA sequences. Numbers at branch points are percentages of bootstrap replicates in which clusters were found. Bars represent the sequence differences among isolates. ${ }^{2}$ Based on data from Poussier et al. (21) and Taghavi et al. (27). B, Phylogenetic tree based on a comparison of the partial endoglucanase gene sequences. C, Phylogenetic tree based on a comparison of the partial $h r p B$ gene sequences. D, Phylogenetic tree based on a comparison of the partial mutS gene sequences. The trees (B, C, and D) were constructed by the neighbor-joining method. Values at branches indicate percent bootstrap support for 1,000 replicates. Bars indicate one nucleotide change per 100 nucleotide positions. ${ }^{b} \mathrm{Based}$ on data from Fegan and Prior (7) and Prior and Fegan (23). 
were divided into two distinct groups, which belonged to phylotype II broad host range (bhr) and phylotype II narrow host range (nhr). The phylotype II bhr group included biovar 1 isolates from Martinique (CFBP2972) and Burkina Faso (CFBP712), and the phylotype II nhr group included biovar 1 isolates from Peru (UW162) and Costa Rica (UW9) and biovar 2 isolates from the Netherlands (CFBP3858) and Reunion Island (JT516; Table 3, Fig. 4B).

The partial $h r p B$ gene sequences, consisting of 1,049 bases covering approximately $75 \%$ of the entire gene, of the 8 isolates were compared to published sequences of 14 reference isolates (Table 3). The phylogenetic tree was divided into four clusters (Fig. 4C). Cluster I, which included 6 Korean isolates (SL2249, SL341, SL2025, TOW3, POW57, and TW56), 2 Japanese isolates (N6211 and MAFF211266), a Malaysian isolate (NCPPB3190), and a Guyanese isolate (GMI1000), belonged to phylotype I (Table 3, Fig. 4C). The cluster II isolates included biovar 1 African isolates (NCPPB1018 and CFBP734) corresponding to phylotype III (Table 3, Fig. 4C). The cluster III isolates, composed of Korean biovar 2 isolate SL2029, Indonesian biovar 2 isolate R142, and the Japanese biovar N2 isolates MARR301558 and MAFF301559, belonged to phylotype IV (Table 3, Fig. 4C). The cluster IV isolates included the phylotype II bhr group, which included biovar 1 isolates from Martinique (CFBP2972) and Burkina Faso (CFBP712), and the phylotype II nhr group, which included biovar 1 isolates from Peru (UW162) and Costa Rica (UW9), as well as biovar 2 isolates from the Netherlands (CFBP3858) and Reunion Island (JT516; Table 3, Fig. 4C).

A phylogenetic tree was generated by comparing 694 nucleotides from the partial $m u t S$ gene sequences of the 8 isolates and compared to the published sequences of 12 isolates (Table 3). Consistent with our analysis of the $h r p B$ gene, the tree was divided into four clusters (Fig. 4D). The cluster I isolates, which belonged to phylotype I, included the Korean biovar 1 isolate SL2249, the Korean biovar 3 isolates SL341, SL2025, and TOW3, the Korean biovar 4 isolates POW57 and TW56, the Japanese biovar 4 isolates N6211 and MAFF211266, a Malaysian isolate (biovar 3: NCPPB3190), and a Guyanese isolate (biovar 3: GMI1000; Table 3, Fig. 4D). The cluster II isolates, which included the African biovar 1 isolates NCPPB1018 and CFBP734, corresponded to phylotype III (Table 3, Fig. 4D). The cluster III isolates were composed of a Korean biovar 2 isolate (SL2029) and a Japanese biovar N2 isolate (MARR301558) and belonged to phylotype IV (Table 3, Fig. 4D). The cluster IV isolates were composed of the phylotype II bhr group, including biovar 1 isolates from Martinique (CFBP2972) and Burkina Faso (CFBP712), and the phylotype II nhr group, including biovar 1 isolates from Peru (UW162) and Costa Rica (UW9), as well as biovar 2 isolates from the Netherlands (CFBP3858) and Reunion Island (JT516; Table 3, Fig. 4D).

\section{DISCUSSION}

Analysis of 109 representative Korean isolates and 16 foreign isolates of $R$. solanacearum by AFLP indicated the existence of two major groups. Among the Korean isolates, six AFLP clusters were revealed, but it is not clear if the genetic diversity among the isolates depends on their geographical origin, host, or biovar. Some inconsistencies were revealed in the groupings when biovar was considered. In division I, biovar 4 was found in all of the clusters except cluster $\mathrm{V}$, which indicates that biovar 4 is the most common biovar among the Korean isolates. The only consistent group in our AFLP analysis was cluster $\mathrm{V}$ in division $\mathrm{I}$, which included all of the Korean biovar 2 (race 3) isolates. Because all of the foreign biovar 2 isolates were part of division II, it appears that the Korean biovar 2 isolates are different from those of foreign origin. This indicates that biovar is useful for analyzing genetic diversity among $R$. solanacearum isolates, but that it cannot be used to distinguish biovar 2, which is consistent with previous observations $(21,22)$.

Our AFLP analysis was further validated by $16 \mathrm{~S}$ rRNA, endoglucanase, $h r p B$, and $m u t S$ gene sequence analyses using 7 Korean and 1 Japanese isolate, and our results support the classification system proposed by Poussier et al. (21), Fegan and Prior (7), and Prior and Fegan (23). Phylogenetic analysis of $16 \mathrm{~S}$ rRNA gene sequences revealed that the Korean biovar 1, 3, and 4 isolates belonged to division 1 , while the Korean biovar 2 isolate belonged to subdivision $2 \mathrm{~b}$, as suggested by Poussier et al. (21). The nucleotides at positions 458 to 460 of the 16S rRNA gene may be useful for distinguishing biovar 2, although there are exceptions. Phylogenetic trees based on the three DNA regions (endoglucanase, $h r p B$, and mutS gene) consistently divided the isolates of $R$. solanacearum into four clusters. The cluster distribution of the isolates also corresponds to the phylotype grouping described by Fegan and Prior (7) and Prior and Fegan (23). The Korean biovar 1, 3, and 4 isolates used in this study belonged to phylotype I, while the Korean biovar 2 isolate belonged to phylotype IV. Taken together, our AFLP, 16S rRNA, endoglucanase, hrpB, and mutS gene sequence analyses indicate that biovar 2 isolates are genetically distinct from the other biovars and have a close genetic relationship with the Japanese biovar N2 isolates.

According to Cook and Sequeira (6), the Americanum division includes biovars 1 ,
2, and N2, while the Asiaticum division contains biovars 3, 4, and 5; however, our analysis showed that the Korean isolates included biovars 1, 2, 3, and 4, suggesting that the Asiaticum division should be revised to include other biovars. Interestingly, the Korean and Japanese isolates belonging to biovars $1,2,3$, and 4 exhibited weak genetic relationships with the American, African, and European isolates, indicating that the genetic backgrounds of the Korean isolates are distinct from those of the foreign isolates used in this study. In addition, since a biovar N2 isolate was not observed among the Korean isolates, it is possible that biovar N2, which is believed to have originated in tropical regions, may not yet be present in Korea.

Among the Korean samples, biovar 2 isolates were found only in a cultivated tomato and potato field in the south. Because biovar 2 isolates are limited to the southern part of Korea and were grouped in a single cluster by AFLP, $16 \mathrm{~S}$ rRNA, endoglucanase, $h r p B$, and mutS gene sequence analyses, it is possible that those isolates might have originated in foreign countries. Buddenhagen (2) suggested that race 3 (biovar 2) originated in the Andean region of South America, and that the occurrence of the race outside of South America is a result of the distribution of infected potato tubers by international trade. There is no record of the existence of biovar 2 isolates in Korea before the potato cultivar Daeji was imported from Japan between 1992 and 2002 (1). It is not obvious from where the Korean biovar 2 isolate originated; however, considering that Daeji is the only potato cultivar grown in southern Korea, the biovar 2 isolates might have arrived with imported potato tubers. To address this possibility, additional Japanese isolates may be needed for a more comprehensive analysis.

\section{ACKNOWLEDGMENTS}

This study was supported by the Technology Development Program for Agriculture and Forestry by the Ministry of Agriculture and Forestry, and the Crop Functional Genomics Center of the 21st Century Frontier R\&D Program, which is funded by Ministry of Science and Technology of the Republic of Korea.

\section{LITERATURE CITED}

1. Anonymous. 1991-2005. Trade in the agriculture, forestry and fishery sector. In: Agricultural and Forestry Statistical Yearbook. Ministry of Agriculture and Forestry, Republic of Korea.

2. Buddenhagen, I. 1985. Bacterial wilt revisited. Pages 126-143 in: Bacterial Wilt Disease in Asia and the South Pacific. G. J. Persley, ed. Aust. Cent. Int. Agric. Res. Proc. 13. Canberra, Australia.

3. Carney, B., and Denny, T. P. 1990. A cloned avirulence gene from Pseudomonas solanacearum determines incompatibility on Nicotiana tabacum at the host species level. J. Bacteriol. 172:4836-4843.

4. Cook, D., Barlow, E., and Sequeira, L. 1989. Genetic diversity of Pseudomonas solanacearum: Detection of restriction fragment length polymorphism with DNA probes that specify 
virulence and the hypersensitive response. Mol. Plant-Microbe Interact. 2:113-121.

5. Cook, D., Barlow, E., and Sequeira, L. 1991. DNA probes as tools for the study of hostpathogen evolution: The example of Pseudomonas solanacearum. Pages 103-108 in: Advances in Molecular Genetics of PlantMicrobe Interactions. Vol. 1. H. Henneke and D. P. S. Verma, eds. Kluwer Academic Publishers, Dordrecht, Netherlands.

6. Cook, D., and Sequeira, L. 1994. Strain differentiation of Pseudomonas solanacearum by molecular genetic methods. Pages 77-93 in: Bacterial Wilt: The Disease and its Causative Agent, Pseudomonas solanacearum. A. C. Hayward and G. L. Hartman, eds. CAB International, Wallingford, UK.

7. Fegan, M., and Prior, P. 2005. How complex is the Ralstonia solanacearum species complex? Pages 449-461 in: Bacterial Wilt Disease and the Ralstonia solanacearum Species Complex. C. Allen, P. Prior, and A. C. Hayward, eds. American Phytopathological Society, St. Paul, $\mathrm{MN}$.

8. Fegan, M., Taghavi, M., Sly, L. I., and Hayward, A. C. 1998. Phylogeny, diversity and molecular diagnostics of Ralstonia solanacearum. Pages 19-33 in: Bacterial Wilt Disease: Molecular and Ecological Aspects. P. Prior, C. Allen, and J. Elphinstone, eds. INRA Edition, Paris.

9. Hayward, A. C. 1964. Characteristics of Pseudomonas solanacearum. J. Appl. Bacteriol. 27:265-277.

10. Hayward, A. C. 1991. Biology and epidemiology of bacterial wilt caused by Pseudomonas solanacearum. Annu. Rev. Phytopathol. 29:6587.

11. He, L. Y., Sequeira, L., and Kelman, A. 1983. Characteristics of strains of Pseudomonas solanacearum. Plant Dis. 67:1357-1361.

12. Horita, M., and Tsuchiya, K. 2000. Comparative analysis of Japanese and foreign strains of Ralstonia solanacearum based on $16 \mathrm{~S}$ ribosomal RNA gene sequences. J. Gen. Plant Pathol. 66:132-137.

13. Horita, M., and Tsuchiya, K. 2001. Genetic diversity of Japanese strains of Ralstonia solanacearum. Phytopathology 91:399-407.
14. Jeanmougin, F., Thompson, J. D., Gouy, M., Higgins, D. G., and Gibson, T. J. 1998. Multiple sequence alignment with Clustal X. Trends Biochem. Sci. 23:403-405.

15. Jeong, Y., Kim, J., Kim, S., Kang, Y., Nagamatsu, T., and Hwang, I. 2003. Toxoflavin produced by Burkholderia glumae causing rice grain rot is responsible for inducing bacterial wilt in many field crops. Plant Dis. 87:890895.

16. Jukes, T. H., and Cantor, C. R. 1969. Evolution of protein molecules. Pages 21-132 in: Mammalian Protein Metabolism. H. N. Munro, ed. Academic Press, New York.

17. Kelman, A. 1954. The relationship of pathogenicity in Pseudomonas solanacearum to colony appearance on a tetrazolium medium. Phytopathology 44:693-695.

18. Kumar, S., Tamura, K., and Nei, M. 2004. MEGA3: Integrated software for molecular evolutionary genetics analysis and sequence alignment. Brief. Bioinform. 5:150-163.

19. Li, X., Dorsch, M., Del Dot, T., Sly, L. I., Stackebrandt, E., and Hayward, A. C. 1993. Phylogenetic studies of the rRNA group II pseudomonads based on 16S rRNA sequences. J. Appl. Bacteriol. 74:324-329.

20. Poussier, S., Prior, P., Luisetti, J., Hayward, A. C., and Fegan, M. 2000. Partial sequencing of the $\operatorname{rrp} B$ and endoglucanase genes confirms and expands the known diversity within the Ralstonia solanacearum species complex. Syst. Appl. Microbiol. 23:479-486.

21. Poussier, S., Trigalet-Demery, D., Vandewalle, P., Goffinet, B., Luisetti, J., and Trigalet, A. 2000. Genetic diversity of Ralstonia solanacearum as assessed by PCR-RFLP of the hrp gene region, AFLP and 16S rRNA sequence analysis, and identification of an African subdivision. Microbiology 146:1679-1692.

22. Poussier, S., Vandewalle, P., and Luisetti, J. 1999. Genetic diversity of African and worldwide strains of Ralstonia solanacearum as determined by PCR-restriction fragment length polymorphism analysis of the hrp gene region. Appl. Environ. Microbiol. 65:2184-2194.

23. Prior, P., and Fegan, M. 2005. Recent developments in the phylogeny and classification of Ralstonia solanacearum. Acta Hortic.
695:127-136

24. Sambrook, J., Fritsch, E. F., and Maniatis, T 1989. Molecular Cloning: A Laboratory Manual. 2nd ed. Cold Spring Harbor Laboratory, Cold Spring Harbor, New York.

25. Seal, S. E., Jackson, L. A., and Daniels, M. J. 1992. Use of tRNA consensus primers to indicate subgroups of Pseudomonas solanacearum by polymerase chain reaction amplification. Appl. Environ. Microbiol. 58:3759-3761.

26. Smith, J. J., Offord, L. C., Holderness, L. C., Holderness, M., and Saddler, G. S. 1995. Genetic diversity of Burkholderia solanacearum race 3 in Kenya. Appl. Environ. Microbiol. 61:4263-4268.

27. Taghavi, M., Hayward, C., Sly, L. I., and Fegan, M. 1996. Analysis of the phylogenetic relationships of strains of Burkholderia solanacearum, Pseudomonas syzygii, and the blood disease bacterium of banana based on 16S rRNA gene sequence. Int. J. Syst. Bacteriol. 46:10-15.

28. Villa, J. E., Tsuchiya, K., Horita, M., Natural, M., Opina, N., and Hyakumachi, M. 2005. Phylogenetic relationships of Ralstonia solanacearum species complex strains from Asia and other continents based on 16S rDNA, endoglucanase, and $h r p B$ gene sequences. J. Gen Plant Pathol. 71:39-46.

29. Vos, P., Hogers, R., Bleeker, M., Reijans, M. van de Lee, T., Hornes, M., Frijters, A., Pot, J. Peleman, J., Kuiper, M., and Zabeau, M. 1995. AFLP: A new technique for DNA fingerprinting. Nucleic Acids Res. 23:4407-4414.

30. Woese, C. R., Gutell, R., Gupta, R., and Noller, H. F. 1983. Detailed analysis of the higher-order structure of 16S-like ribosomal ribonucleic acids. Microbiol. Rev. 47:621-669.

31. Yun, G. S., Park, S. Y., Kang, H. J., Lee, K. Y., and Cha, J. S. 2004. Contamination level of Ralstonia solanacearum in soil of greenhouses cultivating tomato plants in Chungbuk provnce and characteristics of the isolates. Res. Plant Dis. 10:58-62.

32. Yu, Q., Alvarez, A. M., Moore, P. H., Zee, F., Kim, M. S., de Silva, A., Hepperly, P. R., and Ming, R. 2003. Molecular diversity of Ralstonia solanacearum isolate from ginger in $\mathrm{Ha}-$ waii. Phytopathology 93:1124-1130. 